\title{
Impact of Driver Behaviour on availability of Electric Vehicle stored energy for STOR
}

\author{
Luke Morgan \\ Aston University, National Grid \\ Luke.morgan2@nationalgrid.com
}

\author{
Dani Strickland \\ Aston University \\ d.strickland@aston.ac.uk
}

\author{
Laurence Chittock \\ Aston University \\ chittolm@aston.ac.uk
}

\begin{abstract}
As take up of low carbon vehicles increase, there is interest in using the energy stored in the vehicles to help maintain system frequency through ancillary services on the electricity grid system. Research into this area is generally classed as vehicle-to-grid research. In theory, the energy available from electric vehicles could be directly correlated to the vehicle's state of charge (SoC) and battery capacity during the time the car is parked and plugged in. However, not all the energy in the vehicle may be used, as some capacity is required by the driver for their next journey. As such, this paper uses data captured as part of a large scale electric vehicle trial to investigate the effect of three different types of driver routine on vehicle-to-grid availability. Each driver's behaviour is analysed to assess the energy that is available for STOR, with follow on journey requirements also considered.
\end{abstract}

Index Terms-- Ancillary Services, Electric Vehicles, Frequency Response, Operating Reserve, Vehicle-to-Grid Introduction

\section{VEHICLE TO GRID (V2G)}

Present research into Vehicle to grid applications (V2G) has tended to focus on economic or business models, vehicle technology, or communications and simulations on grid impact. Much of the focus has been in the US where the income for frequency support and regulation has been estimated for a number of different services for which "regulation" is potentially one of the more profitable at $\$ 30$ \$45/MWhr [1-3]. These calculations are typically based on probabilistic equations fitted to example based simulation data to take into account factors such as plug in time and vehicle battery size. Due to the size of the vehicle battery and the requirement to meet contractual arrangements, the number of vehicles plugged in at any time must have an aggregated capacity (the achievable power capacity, APC) greater than the contractual power capacity (CPC). At present a number of methods have been suggested to determine the APC. These include probabilistic methods [4], optimisation using stochastic inventory theory [5], predicted charging profiles of the EVs connected to the building for estimating the V2G capacity [6] and queuing theoretic approach, which has been used for performance analysis in communication networks [7]. All of these focus on a suitable method of determination based on a sample or predicted set of data as opposed to real world data based on actual EV usage and charging and discharging.

Real world data has been used to estimate the available power capacity [8], which suggests that energy available for participation in the STOR market could be significant enough to be considered by the UK Utility and also give financial return to EV owners. The paper looked at the energy that was likely to be available on a large scale by considering the time the vehicles are plugged in and when they are charging or sitting at full charge based on data from the 'CABLED' (Coventry And Birmingham Low Emissions Demonstration) project. This paper, builds on that work by specifically looking at the different behaviour patterns of drivers within the project to investigate how driver behaviour and follow on journey requirements may impact the number of vehicles required to be aggregated to guarantee participation in the UK STOR market.

There has been limited work undertaken to date on the effect of driver behaviour in V2G applications. [9] looked at three different driver behaviour patterns based on commuter cars $(80 \%)$, family cars $(15 \%)$ and taxi's $(5 \%)$ using nonlinear state dependent models; however, there was no clear validation of their models against real world data. Other work has focused on the behaviour of drivers including a validated driver grading mechanism to capture the driver behaviour influence on the energy consumption of the vehicle [10] and various accident reduction behavioural studies [11], but unfortunately these methods of classifying driver behaviour do not offer insight into available power capability for grid applications.

This paper looks in more detail at how driver charging and discharging behaviour affects the availability and quantity of energy for STOR use. The work also includes the necessity to account for follow on journey requirements to minimise the risk of the driver being stranded. Three distinct electric vehicle driving records are used to illustrate different real world driver behaviours during the autumn-winter period. A four month autumn-winter period was chosen as this coincides with the peak load period on the transmission system. Not only is the transmission system at its most vulnerable during the winter months - but the energy requirements of an electric vehicle are likely to be higher, due to the increased use of battery-draining ancillaries, such as heating in the car.

The data is obtained from the 'CABLED' (Coventry and Birmingham Low Emissions Demonstration) project which was commissioned in 2009 to showcase and demonstrate the use of ultra-low carbon vehicles (ULCV) in the West Midlands region. Over $100 \mathrm{EVs}$ were leased to members of the public or fleet operators for a minimum period of 12 months, with data collected over a 27 month period. Each 
vehicle in the trial was fitted with a GPS data logger, which recorded the usage, location and charging habits of each vehicle. Every owner had a home charger, and about half had a workplace charger, giving the driver the capability to follow different charging patterns. These vehicles were not used for V2G purposes, so behaviours are based on journey needs/charging convenience only.

It is important that the usage patterns of STOR are studied along with user behaviours in order to form a complete analysis of the potential relationship between the two. Data from all the STOR units connected to the grid has been studied over the same seasonal period as the user behavioural analysis. This data contains the power output of each and every unit as well as the time of day it was utilised. This can be used to determine an interaction between how STOR is despatched and the concept of using consumer electric vehicles as part of this service.

\section{DRIVER BEHAVIOUR}

In order to quantify the benefits of harnessing energy from electric vehicles it is important to be able to predict their availability, much in the same way other sources are predicted, e.g. wind power. This will help form the framework needed to prove the technology can meet the requirements needed to offer response on the transmission system.

Over the whole trial, the homogenised average indicates that the users plug their vehicles in with about 52\% (11KWh) of its energy still available. The vehicles are then charged on average for around 3 hours where they are then either unplugged or left plugged in fully charged. The states where the vehicles are plugged in are obviously the ones of interest in regards to balancing the transmission system. This paper looks more in detail at variation in individual use.

Three different behaviour patterns were chosen in this paper to illustrate the variable behaviour of some EVs within the trial data. There are two different aspects to vehicle charging and discharging behaviour; how often the vehicle is unplugged, and when the vehicle is unplugged and therefore unavailable for grid services. The following three vehicles are representative of three different types of driver behaviour and the information presented on each shows an overall summary of behaviour and more detail on the \% of time the vehicle is plugged in and charging, plugged in and charged and unplugged.

\section{A. Vehicle A}

A summary of the overall charging and discharging for Vehicle A over the four month period is shown in Table I. Fig 1. shows the percentage of time the vehicle was plugged in and unplugged over the study period. This vehicle is typically unplugged overnight, usually plugging in from about 08:00 and then disconnecting at around 18:00. The time the electric vehicle battery is connected to the grid is represented by the blue and red (charging and charged but plugged in) areas on the graph. This gives the impression the vehicle is likely being used as a means of getting to and from work where there is a charging facility available at their workplace. The average available energy from Vehicle $A$ is shown in Fig 2 which is calculated by considering the average available state of charge (minus the $13.2 \%$ which represents an average follow on journey of $6.2 \%+2$ standard deviations (STD) of $7 \%$ ) when the vehicle is plugged in multiplied by the probability that the vehicle is plugged in. The majority of the energy available to the network in this case is in the late afternoon, peaking just under $5 \mathrm{KWh}$.

TABLE I

4 MONTH STATISTICS FOR VEHICLE A

\begin{tabular}{|l|l|}
\hline \multicolumn{2}{|c|}{ Vehicle: $A$} \\
\hline Number of Charges & 31 \\
\hline Average SOC at Start of Charge & $38 \%$ \\
\hline Average Charge Time & $03: 43: 58$ \\
\hline Average SOC Increase & $44.6 \%$ \\
\hline Number of Journeys & 221 \\
\hline Average Journey Duration & $\mathbf{0 0 : 1 5 : 0 5}$ \\
\hline Average SOC Decrease & $6.2 \%$ \\
\hline STD of SOC Decrease & $3.5 \%$ \\
\hline
\end{tabular}

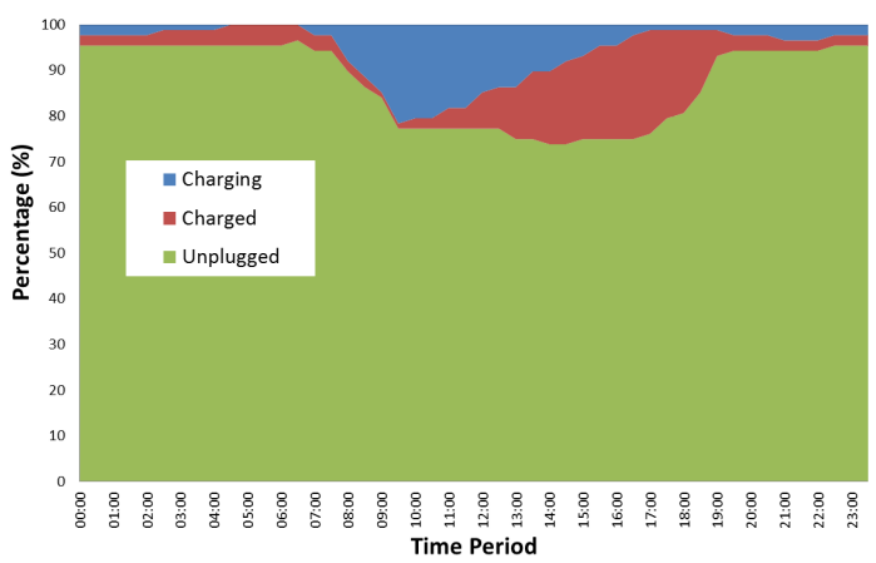

Fig. 1. Vehicle A analysis of data over a four month time span showing a pattern of charging the vehicle during the day but leaving the vehicle unplugged for significant periods of time.

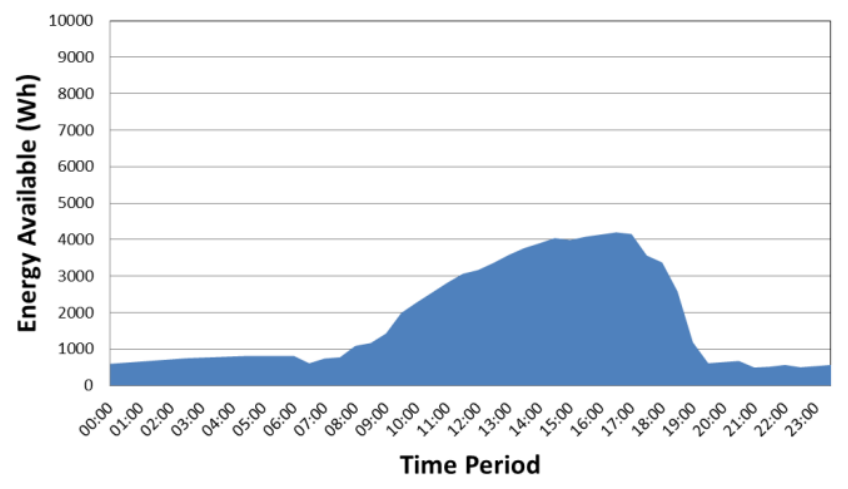

Fig. 2. Vehicle A - Average energy available with time (taking into account follow on journey requirements equal to the average +2 STD) 


\section{B. Vehicle $B$}

A summary of the overall charging and discharging for Vehicle B over the four month period is shown in Table II. The plug in pattern shown below for Vehicle B is very different to the previous pattern shown by Vehicle $\mathrm{A}$ as shown in Fig 3. The availability of this vehicle is quite close to an inverse image of the previous where the availability drops off between typical working hours, picking back up in between. This shows the driver likely uses the vehicle for travelling to work but has no means of charging when there, or chooses not to.

TABLE II

4 MONTH STATISTICS FOR VEHICLE B

\begin{tabular}{|l|c|}
\hline \multicolumn{2}{|c|}{ Vehicle: B } \\
\hline Number of Charges & 27 \\
\hline Average SOC at Start of Charge & $47.4 \%$ \\
\hline Average Charge Time & $04: 03: 32$ \\
\hline Average SOC Increase & $43.1 \%$ \\
\hline Number of Journeys & 104 \\
\hline Average Journey Duration & $00: 20: 41$ \\
\hline Average SOC Decrease & $13 \%$ \\
\hline STD of SOC Decrease & $12 \%$ \\
\hline
\end{tabular}

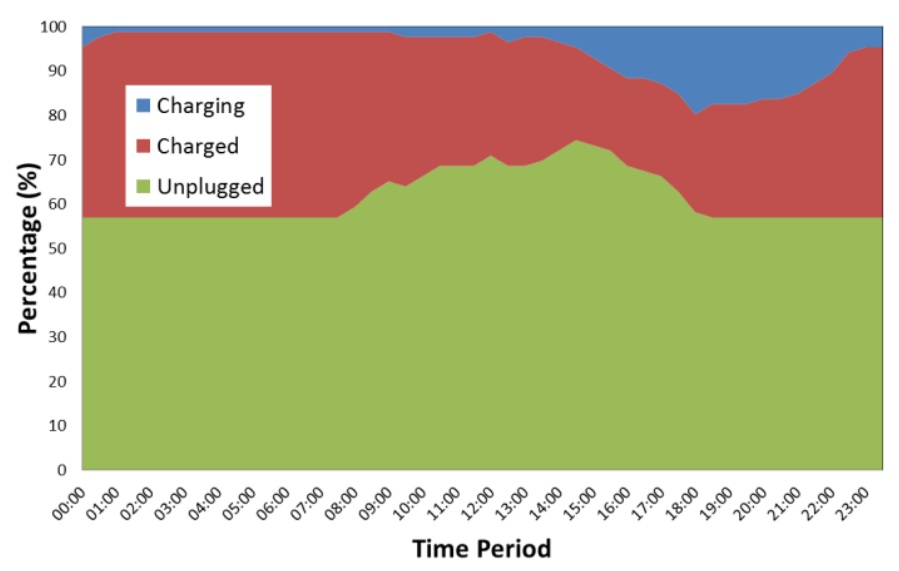

Fig. 3. Vehicle B analysis of data over a 4 month time span showing a pattern of charging the vehicle overnight and leaving it plugged in.

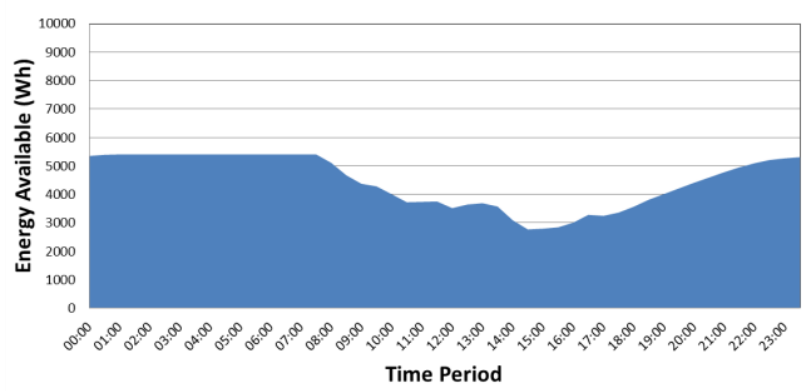

.Fig. 4. Vehicle B - Average energy available with time (taking into account follow on journey requirements equal to the average $+2 \mathrm{STD}$ )

The data indicates that the driver leaves their vehicle plugged in even after it has reached full charge. The average journey uses $10.7 \%(2284 \mathrm{Wh})$ of the battery capacity, with the typical duration time being just over 20 minutes long. The energy available for the grid peaks at under $8 \mathrm{kWh}$ and dips to $4 \mathrm{kWh}$ during peak time.

\section{Vehicle C}

A summary of the overall charging and discharging for Vehicle $\mathrm{C}$ over the four month period is shown in Table III. The plug in pattern shows that the user charges their vehicle frequently and uses the vehicle rarely although the pattern of charging is similar to Vehicle B. The plugged and unplugged status of the vehicle can be seen in Fig 5 along with the energy available in Fig 6.

TABLE III

4 MONTH STATISTICS FOR VEHICLE C

\begin{tabular}{|l|c|}
\hline \multicolumn{2}{|c|}{ Vehicle: C } \\
\hline Number of Charges & $\mathbf{5 6}$ \\
\hline Average SOC at Start of Charge & $\mathbf{7 7 . 3 \%}$ \\
\hline Average Charge Time & $\mathbf{0 1 : 3 3 : 1 5}$ \\
\hline Average SOC Increase & $\mathbf{1 6 . 9 \%}$ \\
\hline Number of Journeys & $\mathbf{1 7 4}$ \\
\hline Average Journey Duration & $\mathbf{0 0 : 1 6 : 0 0}$ \\
\hline Average SOC Decrease & $\mathbf{5 . 5 \%}$ \\
\hline STD of SOC Decrease & $\mathbf{5 \%}$ \\
\hline
\end{tabular}

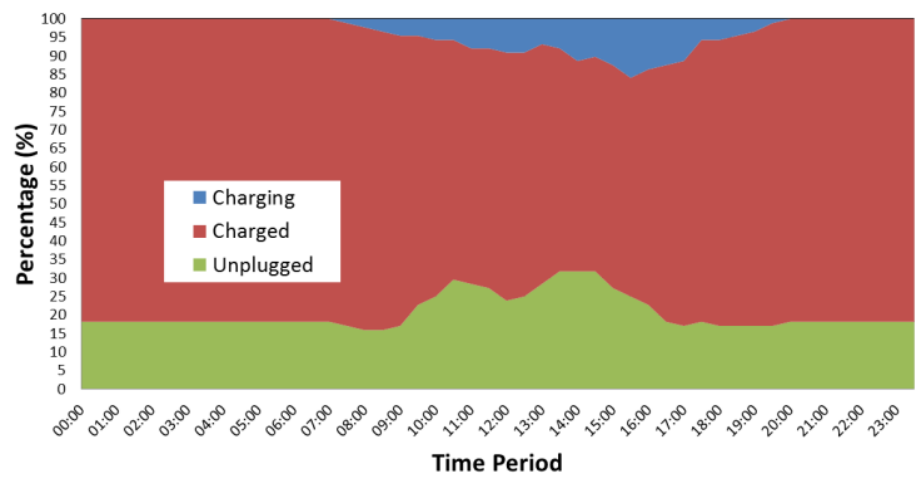

Fig. 5. Vehicle $\mathrm{C}$ analysis of data over a four moth time span showing a pattern low usage and leaving the vehicle plugged in.

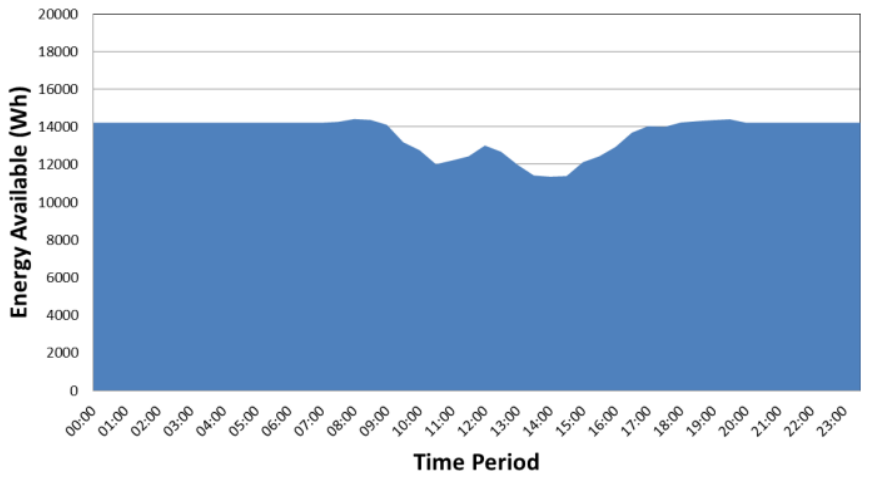

Fig. 6. Vehicle $\mathrm{C}-$ Average energy available with time (taking into account follow on journey requirements equal to the average $+2 \mathrm{STD}$ )

\section{Summary of behaviour types}

The different driver behaviour types have been loosely classified in Table IV. This allows each individual behaviour type to be classified by both time of charging and availability 
of vehicle. A more in-depth analysis of each vehicle is needed to fully understand if this is the most appropriate split. The majority of the fleet charging behaviour is most similar to that of Driver B. However, it is apparent that greater analysis is needed to understand the variations of use with these generalised behaviour patterns. In particular it is necessary to understand the effect of weekday/weekend, holidays and seasonal differences on this behaviour pattern.

TABLE IV

DIFFERENT CHARGING AND DISCHARGING BEHAVIOUR

\begin{tabular}{|l|l|c|c|}
\hline Type & $\begin{array}{l}\text { V2G } \\
\text { availability }\end{array}$ & $\begin{array}{c}\text { Majority } \\
\text { time of } \\
\text { charge }\end{array}$ & $\begin{array}{l}\text { Average \% } \\
\text { charge } \\
\text { available }\end{array}$ \\
\hline 1 (Car A) & Low & Day & $\mathbf{0 - 2 0 \%}$ \\
\hline $\mathbf{2}$ & Low & Night & $\mathbf{0 - 2 0 \%}$ \\
\hline $\mathbf{3}$ & Med & Day & $\mathbf{2 0 - 5 0 \%}$ \\
\hline $\mathbf{4}($ Car B) & Med & Night & $\mathbf{2 0 - 5 0 \%}$ \\
\hline $\mathbf{5}$ & High & Day & $>\mathbf{5 0 \%}$ \\
\hline 6 $($ Car C) & High & Night & $>\mathbf{5 0 \%}$ \\
\hline
\end{tabular}

\section{STOR}

Short Term Operating Reserve (STOR) is a contracted balancing service used by National Grid in the UK. It offers financial incentives for both generation and demand increase and/or reduction and is used when there is an unexpected shortfall in matching generation with demand. This service is managed using bespoke contracts between the providers and National Grid [12].

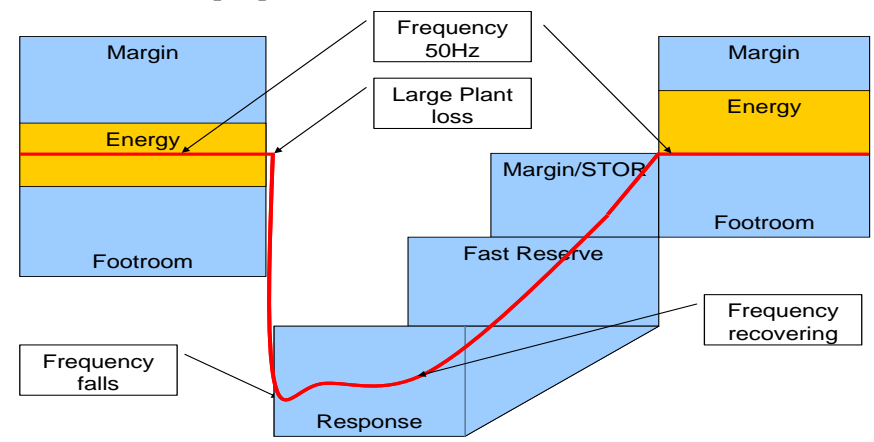

Fig. 7. National Grid Response and Reserve Management showing how STOR response fits into the toolbox of frequency control available to National Grid [12].

The use of STOR in regards to frequency is shown graphically in Figures 7 and 8. National Grid has to operate within statutory limits of between $49.5 \mathrm{~Hz}$ and $50.5 \mathrm{~Hz}$ which are deemed to be acceptable limits as detailed in the SQSS (Security and Quality of Supply Standards) [13]. However, internal policy is to aim to try and keep the frequency within tighter operational limits of between $49.8 \mathrm{~Hz}$ and $50.2 \mathrm{~Hz}$.

If an incident occurs on the system such as a large generation loss the frequency will fall and it is important this is resolved as quickly as possible. There are a number of response mechanisms which are used to respond to this with the fast STOR operating within 5-20 minutes time slot.

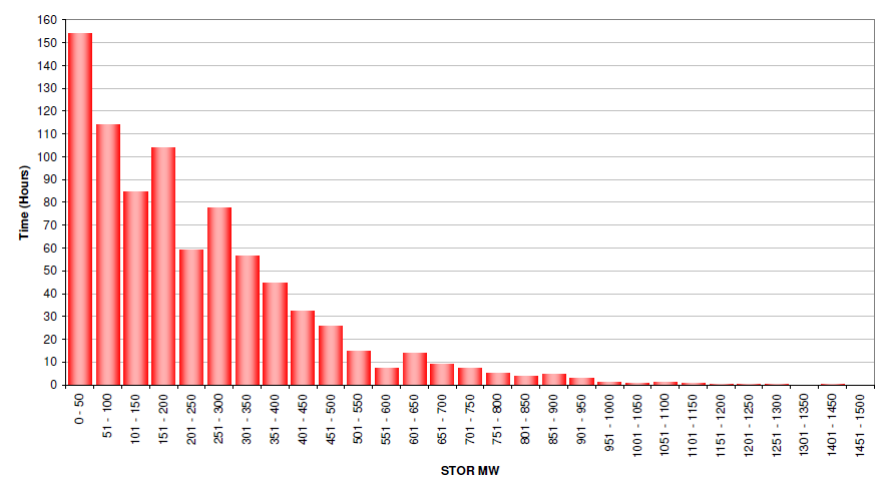

Fig. 8. National Grid STOR versus time commitments for 2012-2013. This shows that the most frequently contracted units are those <50MW which is where aggregated V2G usage would presume to lie.

To provide the STOR service there are a number of contractual requirements which must be met; The provider must be able to supply a minimum of $3 \mathrm{MW}$ of output power, the service must be available at least 3 times a week, have a recovery period after provision of reserve of not more than 1200 minutes, and they must be able to maintain their contracted output for at least 2 hours. There are two types of response time for providers which are short notice and long notice. Long notice providers are available in more than 20 minutes and less than 4 hours from request, and short notice providers are available within 20 minutes of instruction. Short notice is the preferable response time for STOR and the notice period that V2G batteries should fall under.

There are two forms of STOR service, a committed service or a flexible service. A committed service offers service availability in all required windows in each season which National Grid are obliged to buy. This service can be offered by BMU (Balancing Mechanism Unit) and non-BMU providers.

The flexible service option is one which has no obligation to supply services at a certain time along with no obligation for National Grid to accept services offered, thus offering a flexible STOR service for both parties. This service can only be tendered for by non-BMU providers. Out of the two types of service available (Committed and Flexible) the flexible service is more suitable for Vehicle to Grid providers. This type of service does not require rigid availability like the committed service and caters to the unpredictability of user behaviour.

This paper investigates more closely the relationship between electric vehicles as a provider of STOR services under these conditions taking into account driver behaviour by comparing how the vehicles may be made to match the published STOR services over a winter period. Fig 8 shows the size of the contracted STOR service against the frequency of usage over the 2012 and 2013 period. The STOR service has also been looked at more closely on a daily basis with typical weekly contracted figures shown in Table V. 
TABLE V

EXAMPLE OF A WEEKLY CONTRACTED STOR SERVICE

\begin{tabular}{|l|l|}
\hline \multicolumn{2}{|c|}{ Total Utilised Volume (MWhr) } \\
\hline Monday & 31577 \\
\hline Tuesday & 24281 \\
\hline Wednesday & 19589 \\
\hline Thursday & 18897 \\
\hline Friday & 23179 \\
\hline Saturday & 27484 \\
\hline Sunday & 22217 \\
\hline
\end{tabular}

These figures show that Mondays are when the service is used most. This is thought to be due to the fact that Sundays are a low demand day and more generators come on to the system cold on the Monday. This makes them more liable to tripping off the system through faults when synchronising/synchronised to the grid, thus inducing more need for reserve to replace these losses and meet the demand pickups.

The usage then dips for the rest of the week with some moderate changes with a pickup on the Saturday. STOR usage is driven to an extent by forecasting inaccuracies and the pickups on a Saturday are less transparent than the week days. These high level figures have been broken down further to look for any differences in the daily behaviours as shown in Fig. 9. And Fig 10 which show the daily services on a Monday (most heavily contracted) and Thursday (lowest contracted service) over each season. This is to see if there are any issues when coordinating the STOR patterns with that of the electric vehicle battery patterns.

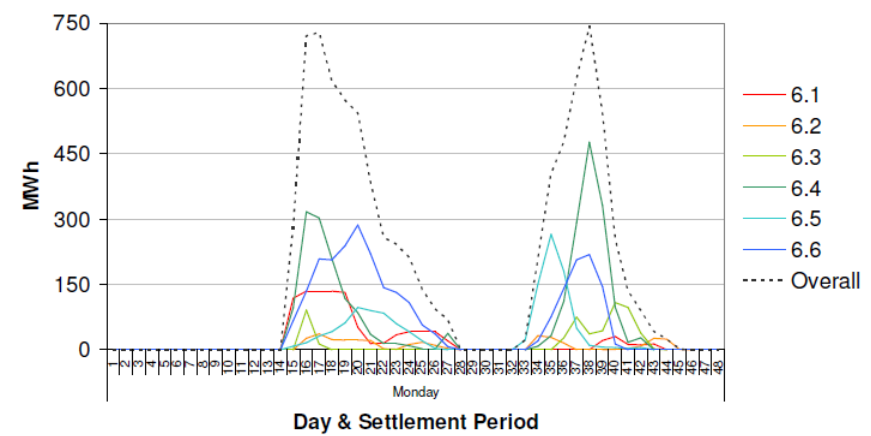

Fig. 9. National Grid STOR example of a commitment on a Monday for each pre-defined season.

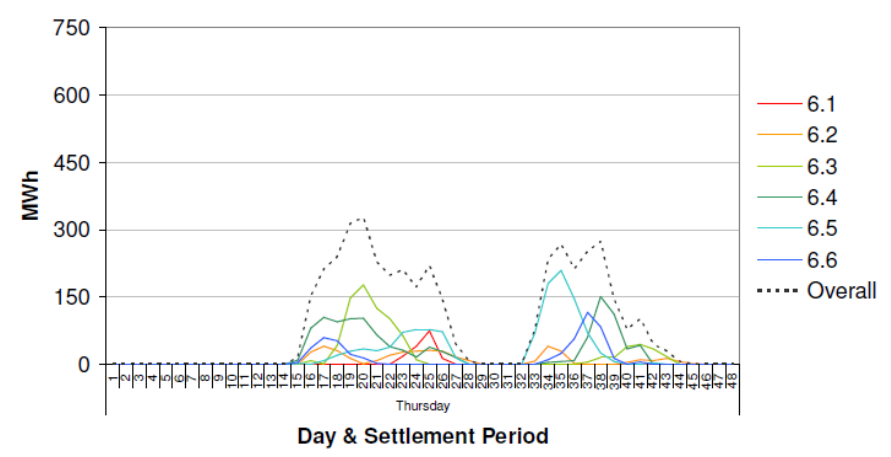

Fig. 10. National Grid STOR example of a commitment on a Thursday.
This shows that the most frequently contracted units are those over morning and afternoon pick up and drop off. The contracted value on a Thursday is lower but the shape is similar. From the 11th November 2013 until the 12th December 2013880 GWh's of STOR was contracted for use on the system, of which only 3\% ( 26.6GWh's) was utilised. This comparison can be seen in Fig 11. and illustrates the large gap between the two contracted and used service level. This information is potentially promising in regards to issues such as maximising vehicle battery life as the STOR requirement is primarily availability with low overall energy usage.

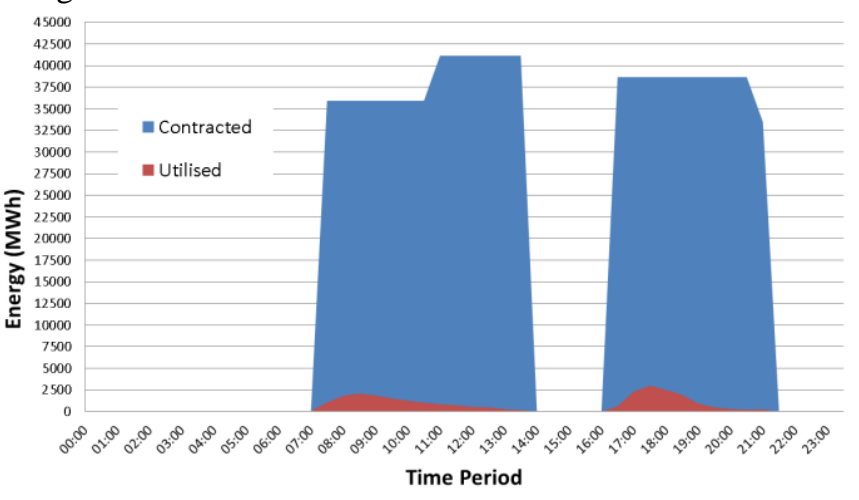

Fig. 11. National Grid aggregated contracted and utilized STOR services as a function of time

Contracting the energy for availability during the required times comes at a cost of approximately $£ 170,000$ a day, or around $£ 6.40$ for each MWh made available. These payments are made regardless of whether the provider is actually used or not which is a huge financial incentive. The energy that is utilised is then charged at whatever the rate is of that provider.

As expected the outputs are highest during the daily demand peaks which STOR is primarily contracted for. This shows the importance of having the vehicle batteries available at these times and can be used to determine just how much energy may be needed and for how long. Accumulatively over the studied period the morning and evening peaks totalled around 4 and 6GWh's respectively.

\section{MATCHING V2G TO STOR}

The time period that the STOR service is required was compared to the number of vehicles of each vehicle type needed in order to achieve a minimum 3MW worth of available energy over each time slot. (using the energy available from Fig 2, 4 and 6). The results of this are illustrated in Fig 12, found by dividing $3 \mathrm{MW}$ by the energy in each $1 / 2$ slot from each vehicle type to get the number of vehicles needed to provide STOR over that time period. The results show that around 6000 vehicles of behaviour type A would be needed to meet the STOR requirement for $3 \mathrm{MW}$ 
support compared to 925 of the same type as Vehicle B and 250 of the same type as Vehicle C. This is mostly due to the time the vehicle is unplugged for travelling coinciding with the peak demand time.

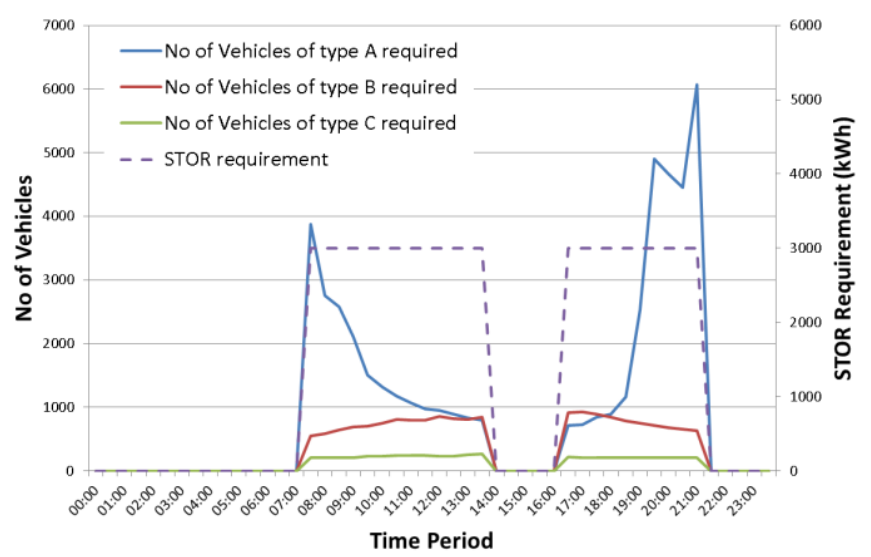

Fig. 12. No of vehicles of each behaviour type needed to meet the STOR requirements.

It is worth noting that 250 low usage vehicles similar to vehicle $\mathrm{C}$ would have a potential aggregated capacity of close to $5 \mathrm{MWh}$ with an approximate value to the vehicle owner (for availability not energy used) of $£ 0.80$ per day based on 11.5 hours of 3MW STOR availability. Vehicles similar to Vehicle $B$ would have an estimated income of $25 \mathrm{p}$ per day and Vehicles similar to Vehicle A would generate $4 p$ per day. There is additional scope to undertake STOR services by reducing demand as well as outputting power. A similar analysis could be used to understand how many vehicles of each type would have to stop charging in order to provide a $3 \mathrm{MW}$ reduction in load using the charging only data from Fig 1, Fig 3 and Fig 5.

\section{CONCLUSION}

STOR is most commonly used at times of rapid demand pick up or very high demand peaks. For instance in winter, the morning pick-up starts at 06:30 peaking at 08:30, whilst the evening pickup starts at 16:00 peaking at 17:30. However, none of the behaviour patterns of the electric vehicles studied ideally match this output. Vehicles that are plugged in during the day time (such as where charging at work is available) are either just plugging in or just disconnecting. Vehicles that are plugged in overnight are typically not available for early evening pick up and their availability in the evening coincides with the need to charge (which could be managed if helpful for network constraints), and varies significantly with time.

Additional work is required to look more closely at daily variation, seasonal variation and the effect of holidays. It is also necessary to understand better the percentage of vehicles of each type and how this is subject to change. This will allow a more detailed analysis of how the vehicles may be best aggregated- potentially in conjunction with the optimisation studies already conducted in the literature. The identification of charging profiles can be used to quantify the benefits of STOR and the expected loss of life from the battery is expected to be low based on the large difference between contracted and utilised energy.

\section{REFERENCES}

[1] W Kempton, "A Test of Vehicle-to-Grid (V2G) for Energy Storage and Frequency Regulation in the PJM System" available online, http://www.udel.edu/V2G/resources/test-v2g-in-pjm-jan09.pdf

[2] Chukwu, U.C. ; Mahajan, S.M. "V2G electric power capacity estimation and ancillary service market evaluation", Power and Energy Society General Meeting, 2011 IEEE pp 1- 8

[3] Ahmad, M.R.; Musirin, I.; Othman, M.M. "Profit determination for vehicle-to-grid (V2G) operation in smart grid environment", Power Engineering and Optimization Conference (PEOCO), 2013 IEEE 7th International, On page(s): $758-763$

[4] Sekyung Han; Soohee Han; Sezaki, K. "Estimation of Achievable Power Capacity From Plug-in Electric Vehicles for V2G Frequency Regulation: Case Studies for Market Participation" Smart Grid, IEEE Transactions on Volume: 2, 2011, pp $632 \quad-641$

[5] Hao Liang; Bong Jun Choi; Weihua Zhuang; Xuemin Shen "Optimizing the Energy Delivery via V2G Systems Based on Stochastic Inventory Theory", Smart Grid, IEEE Transactions on, On page(s): 2230 - 2243 Volume: 4, Issue: 4, Dec. 2013

[6] Kumar, K.N.; Sivaneasan, B.; Cheah, P.H.; So, P.L.; Wang, D.Z.W. "V2G Capacity Estimation Using Dynamic EV Scheduling", Smart Grid, IEEE Transactions on, On page(s): 1051 - 1060 Volume: 5, Issue: 2, March 2014

[7] Lam, A.Y.S.; Ka-Cheong Leung; Li, V.O.K. "Capacity management of vehicle-to-grid system for power regulation services", Smart Grid Communications (SmartGridComm), 2012 IEEE Third International Conference on, On page(s): 442 - 447

[8] M Coldwell, D Strickland, L Chittock "The Impact of Electric Vehicles on Great Britain's Electricity Demand, and the Potential Associated Benefits for System Control", UPEC 2013

[9] Sundstrom, O. ; Binding, C. "Flexible Charging Optimization for Electric Vehicles Considering Distribution Grid Constraints",Smart Grid, IEEE Transactions on , Volume: 3, 2012, pp 26- 37

[10] Bogdan Rosca 1, Steven Wilkins, "On the Sensitivity of the State of Batteries on Driver Behaviour within the EV Powertrain", EEVC, Brussels, Belgium, November 19-22, 2012

[11] Hang-Bong Kang , "Various Approaches for Driver and Driving Behavior Monitoring: A Review",Computer Vision Workshops (ICCVW), 2013 IEEE Int. 2013, pp 616- 623

[12] National Grid, Short Term Operating Reserve General Description of the Service, [Online] Available from: http://www.nationalgrid.com/NR/rdonlyres/083D0D9C-1A33-43368FA3-1A69DCC1C903/61678/TR21_General_Description.pdf [Accessed 15th August 2013]

[13] National Grid, National Electricity Transmission System Security and Quality of Supply Standard, [Online] Available from: http://www.nationalgrid.com/NR/rdonlyres/0F3512E9-EFE9-4A3F8F0F-57FBD67652DA/52977/NETSSQSS version23changemarked1.pdf [Accessed 16th August 2013] 